\title{
A New Method for Obtaining the Baryons Mass under the Killingbeck Plus Isotonic Oscillator Potentials
}

\author{
Nasrin Salehi \\ Department of Basic Sciences, Shahrood Branch, Islamic Azad University, Shahrood, Iran \\ Correspondence should be addressed to Nasrin Salehi; salehi@shahroodut.ac.ir
}

Received 27 May 2016; Revised 11 July 2016; Accepted 21 July 2016

Academic Editor: Mohammadreza Hadizadeh

Copyright ( 2016 Nasrin Salehi. This is an open access article distributed under the Creative Commons Attribution License, which permits unrestricted use, distribution, and reproduction in any medium, provided the original work is properly cited. The publication of this article was funded by SCOAP . $^{3}$

\begin{abstract}
The spectrum of ground state and excited baryons $(N, \Delta, \Lambda, \Sigma, \Xi$, and $\Omega$ particles) has been investigated by using nonrelativistic quantum mechanics under the Killingbeck plus isotonic oscillator potentials. Using the Jacobi coordinates, anzast method, and generalized Gürsey Radicati (GR) mass formula the three-body-wave equation is solved to calculate the different states of the considered baryons. A comparison between our calculations and the available experimental data shows that the position of the Roper resonances of the nucleon, the ground states, and the excited multiplets up to three $\mathrm{GeV}$ are in general well reproduced. Also one can conclude that the interaction between the quark constituents of baryon resonances could be described adequately by using the combination of Killingbeck and isotonic oscillator potentials form.
\end{abstract}

\section{Introduction}

The hadrons spectroscopy is very important to study its structures and the nature of the interacting forces between its constituents. In quark models, the baryons are threequark states and there are number of very different model calculations for the baryon masses [1-8]. Such a picture of these elementary particles has been having success in explaining and describing spatial ground state of the flavor $S U(3)$ vector mesons and baryon octet. But in recent years, baryon spectroscopy has attracted much interest because baryons were the focal point of quark model development $[9,10]$. Such a system can be studied by quantum chromodynamics which describes what between quarks and gluon and their interactions [11, 12].

In order to study massive baryons there are two options, experimentally and theoretically. For example, experimentally mass spectrum of singly charmed heavy baryons is well known but the other charmed heavy baryons are not. Recently, Abazov et al. and Aaltonen et al. have published articles separately in which there are mass measurements of singly bottom baryon $\Xi_{b}^{-}$by $D \emptyset[13]$ and CDF [14] collaborations. On the other hand, from lattice QCD point of view, there are interesting efforts about quenched calculations such as what is done by Bowler et al., in which they presented the results of an exploratory lattice study of heavy baryon spectroscopy [15], or Lewis et al. who calculated masses of singly and doubly charmed baryons in quenched lattice QCD using an improved action of the D234 type on an anisotropic lattice [16] and/or Mathur et al. who computed the mass spectrum of charmed and bottom baryons on anisotropic lattices using quenched lattice nonrelativistic QCD [17]; also Gottlieb and Tamhankar published results from a lattice study of the semileptonic decay of $\Lambda_{b} \rightarrow \Lambda_{c} l \nu_{l}$ [18] and Ali Khan et al. presented lattice results for the spectrum of mesons containing one heavy quark and baryons containing one or two heavy quarks [19], and in this discipline reader can check [20] and for dynamical sea quark flavor simulations, $\mathrm{Na}$ and Gottlieb studied the heavy baryon mass spectrum on gauge configurations that include $2+1$ flavors of dynamical improved staggered quarks [21] and they present results for the mass spectrum of charm and bottom heavy baryons, using MILC coarse lattice configurations with $2+1$ flavors [22]; and also Lewis and Woloshyn bottom calculated baryon masses based on a $2+1$ flavor dynamical lattice QCD simulation. Of course that for the heavy baryon mass spectrum and most results is in fair agreement with observed values [23]. On the other hand motivation of studying light baryons is that 
it enables us to find an understating of the structures and their interactions [24]. Actually to have fundamental manifestation for the long-distance quark and gluon dynamics that is governed in QCD, we use hadron mass spectra. As a matter of fact, nonperturbative calculations and numerical simulation in space-time lattice [25] lead to method to get to this matter from QCD Lagrangian without having any approximations; this results in determination of light quark masses as well [26]. In the recent years considerable effort has been done in the lattice QCD calculation of the light hadron spectrum [2729]. We can mention a calculation in which mass of hadron has been calculated with accuracy of $0.5 \%-3 \%$ [30, 31].

As a matter of fact, the three-quark interaction can be divided in two parts: the first one, containing the confinement interaction, is spin and flavor independent and it is therefore $S U(6)$ invariant, while the second one violates the $S U(6)$ symmetry [1,32-35]. It is well known that Gürsey Radicati mass formula [36] describes quite well the way $S U(6)$ symmetry is broken, at least in the lower part of the baryon spectrum. In this work we applied the generalized Gürsey Radicati (GR) mass formula which is presented by Giannini et al. [8] to calculate the baryon masses. The model we used is a simple Constituent Quark Model in which the $S U(6)$ invariant part of the Hamiltonian is the same as in the hypercentral Constituent Quark Model $[37,38]$ and the SU(6) symmetry is broken by a generalized GR mass formula. In this paper the exact solution of the Schrodinger equation for the Killingbeck plus quantum isotonic oscillator potentials [39-41] via wave function ansatz is given and we introduce the generalized GR mass formula; then we give the results obtained by fitting the generalized GR mass formula parameters to the baryon masses and we compare our calculation spectrum with the experimental data.

\section{The Used Theoretical Model}

The Hamiltonian of the Schrödinger equation is as the following form:

$$
H=\frac{-\hbar^{2}}{2 \mu} \nabla^{2}+V(r)
$$

where

$$
\nabla^{2}=\left[\frac{d^{2}}{d r^{2}}+5 r^{-1} \frac{d}{d r}-\frac{\gamma(\gamma+4)}{r^{2}}\right],
$$

since the Schrödinger equation is

$$
H \psi_{\nu \gamma}=E \psi_{\nu \gamma}
$$

in the six dimensions, these equations for a system containing three quarks with a potential $V(r)$ and by considering $\psi_{\nu \gamma}=$ $u_{\nu \gamma}(r) r^{-5 / 2}$ can be written as

$$
\begin{aligned}
& \frac{d^{2} u_{\gamma \gamma}(r)}{d r^{2}} \\
& \quad+2 \mu\left[E-V(r)-\frac{(2 \gamma+5)(2 \gamma+3)}{8 \mu r^{2}}\right] u_{\gamma \gamma}(r) \\
& \quad 0
\end{aligned}
$$

where $u_{\nu \gamma}(r), r$, and $\gamma$ are the hyperradial wave function, the hyperradius, and the grand angular quantum number, respectively. $\gamma$ is also given by $\gamma=2 n+l_{\rho}+l_{\lambda}, 0 \leq n \leq \infty$ with the angular momenta $l_{\rho}$ and $l_{\lambda}$ which are associated with the Jacobi coordinates $(\vec{\rho}$ and $\vec{\lambda})[44]$ and $\nu$ denotes the number of nodes of three-quark wave functions. In (4) $\mu$ is the reduced mass which is defined as $\mu=m_{\rho} m_{\lambda} /\left(m_{\rho}+m_{\lambda}\right)$ in which $m_{\rho}=$ $m_{1} m_{2} /\left(m_{1}+m_{2}\right), m_{\lambda}=3 m_{3}\left(m_{1}+m_{2}\right) / 2\left(m_{1}+m_{2}+m_{3}\right)$; $m_{1}, m_{2}$, and $m_{3}$ are the constituent quark masses [38]. In our model, the interaction potential is assumed as

$$
V(r)=a r^{2}+b r+\frac{c}{r}+\frac{d}{r^{2}}+\frac{h r}{r^{2}+1}+\frac{k r^{2}}{\left(r^{2}+1\right)^{2}} .
$$

Cornell interaction (Coulomb plus linear) which is static and spherically symmetric interaction has a physical application in Mesonic systems, that is, Charmonium and Bottomonium. Coulomb-like part potential is a short range potential that arises from exchanging a massless gluon between the quarks, whereas linear part is a long range. Coulombic interaction is known from perturbative quantum chromodynamics and the large distance interaction known from lattice QCD [45-47]. We modify the Cornell potential by adding the harmonic term. The resultant quark-antiquark interaction is known as Killingbeck potential which is $a r^{2}+$ $b r+c / r$ [48]. Notice those terms regarding isotonic-type interaction. The energy spectrum of the isotonic potential is isomorphous to the harmonic oscillator spectrum; that is, it consists of an infinite set of equidistant energy levels. For this reason this oscillator is called "the isotonic oscillator." Generalized isotonic oscillators can be seemed as possible representations of realistic quantum dots [41]. The behavior of the Killingbeck plus isotonic oscillator can be seen in Figure 1.

By substituting (5) into (4) we obtain the following equation:

$$
\begin{gathered}
\frac{d^{2} u_{\nu \gamma}(r)}{d r^{2}}=-2 \mu\left[E-a r^{2}-b r-\frac{c}{r}-\frac{d}{r^{2}}-\frac{h r}{r^{2}+1}\right. \\
\left.-\frac{k r^{2}}{\left(r^{2}+1\right)^{2}}-\frac{(2 \gamma+5)(2 \gamma+3)}{8 \mu r^{2}}\right] u_{\nu \gamma}(r) .
\end{gathered}
$$

And regarding $r^{2} /\left(r^{2}+1\right)^{2}=1 /\left(r^{2}+1\right)-1 /\left(r^{2}+1\right)^{2}$ we have

$$
\begin{aligned}
& \frac{d^{2} u_{\nu \gamma}(r)}{d r^{2}}=\left[-2 \mu E+2 \mu a r^{2}+2 \mu b r+2 \mu \frac{c}{r}+2 \mu \frac{d}{r^{2}}\right. \\
& +2 \mu \frac{h r}{r^{2}+1}+2 \mu \frac{k}{r^{2}+1}-2 \mu \frac{k}{\left(r^{2}+1\right)^{2}} \\
& \left.+\frac{(2 \gamma+5)(2 \gamma+3)}{4 r^{2}}\right] u_{\nu \gamma}(r) .
\end{aligned}
$$

We suppose the following form for the wave function:

$$
u_{v \gamma}(r)=g(r) \exp (f(r)) \text {. }
$$




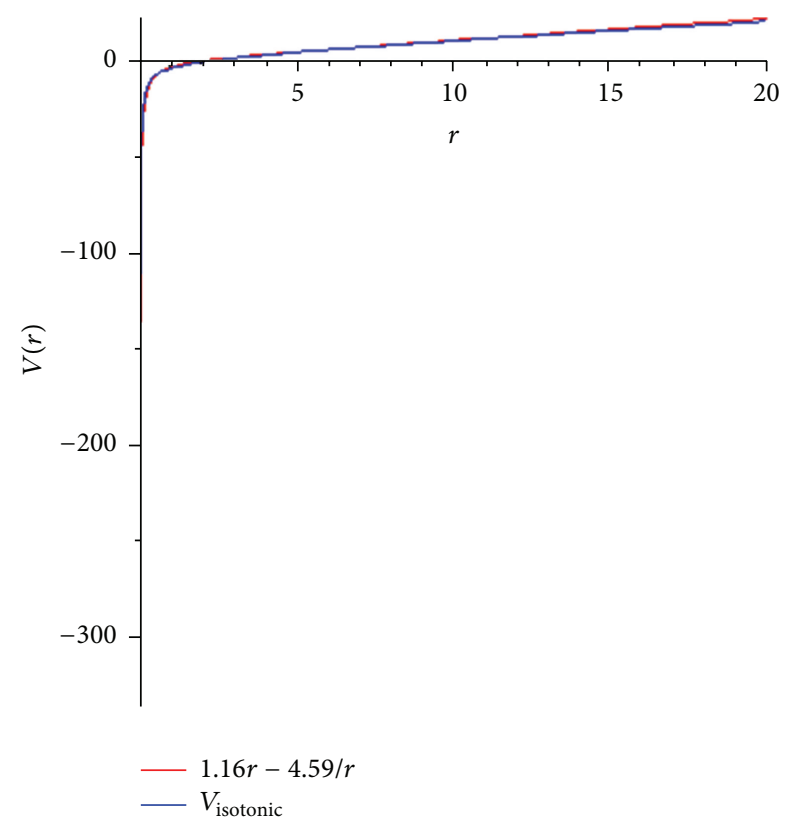

FIgURE 1: The red color shows the Cornell potential and the blue color shows the Killingbeck plus isotonic oscillator potential for $a=$ $0.0072, b=1.16, c=-4.59, d=0.0032, h=-2.7904$, and $k=$ 1.01008 .

Now for functions $f(r)$ and $g(r)$ we make use of the ansatz [49-53]:

$$
\begin{aligned}
& g(r)= \begin{cases}1 & v=0 \\
\prod_{i}^{v}\left(r-\alpha_{i}^{v}\right) & v \geq 1,\end{cases} \\
& f(r)=\alpha r^{2}+\beta r+\lambda \ln r+\eta \ln \left(r^{2}+1\right), \quad \alpha>0 .
\end{aligned}
$$

From (8) we obtain

$$
\begin{aligned}
& u_{\nu, \gamma}^{\prime \prime}(r) \\
& =\left[f^{\prime \prime}(r)+f^{\prime 2}(r)+\frac{2 f(r) g^{\prime}(r)+g^{\prime \prime}(r)}{g(r)}\right] u_{\nu, \gamma}(r) .
\end{aligned}
$$

And from (9) we have

$$
\begin{aligned}
f^{\prime}(r)= & 2 \alpha r+\beta+\frac{\lambda}{r}+\frac{2 \eta r}{\left(r^{2}+1\right)}, \\
f^{\prime 2}(r)= & 4 \alpha^{2} r^{2}+\beta^{2}+4 \alpha \beta r+\frac{\lambda^{2}}{r^{2}}+\frac{4 \eta^{2} r^{2}}{\left(r^{2}+1\right)^{2}} \\
& +\frac{4 \lambda \eta}{r^{2}+1}+4 \alpha \lambda+\frac{8 \alpha \eta r^{2}}{r^{2}+1}+\frac{2 \beta \lambda}{r} \\
& +\frac{4 \beta \eta r}{r^{2}+1}, \\
f^{\prime \prime}(r)= & 2 \alpha-\frac{\lambda}{r^{2}}+2 \eta \frac{\left(r^{2}+1\right)-2 r^{2}}{\left(r^{2}+1\right)^{2}} \\
f^{\prime \prime}(r)= & 2 \alpha-\frac{\lambda}{r^{2}}+\frac{2 \eta}{\left(r^{2}+1\right)}-\frac{4 \eta r^{2}}{\left(r^{2}+1\right)^{2}} .
\end{aligned}
$$

Regarding $r^{2} /\left(r^{2}+1\right)^{2}=1 /\left(r^{2}+1\right)-1 /\left(r^{2}+1\right)^{2}$ and $r^{2} /\left(r^{2}+\right.$ $1)=1-1 /\left(r^{2}+1\right)$ we have

$$
\begin{aligned}
f^{\prime 2}(r)= & 4 \alpha^{2} r^{2}+\beta^{2}+4 \alpha \beta r+\frac{\lambda^{2}}{r^{2}}+\frac{4 \eta^{2}}{r^{2}+1} \\
& -\frac{4 \eta^{2}}{\left(r^{2}+1\right)^{2}}+\frac{4 \lambda \eta}{r^{2}+1}+4 \alpha \lambda+8 \alpha \eta \\
& -\frac{8 \alpha \eta}{r^{2}+1}+\frac{2 \beta \lambda}{r}+4 \beta \eta \frac{r}{r^{2}+1}, \\
f^{\prime \prime}(r)= & 2 \alpha-\frac{\lambda}{r^{2}}-\frac{2 \eta}{r^{2}+1}+\frac{4 \eta}{\left(r^{2}+1\right)^{2}} .
\end{aligned}
$$

Substituting (11) and (12) into (10) leads to

$$
\begin{aligned}
& u_{\nu \gamma}^{\prime \prime}(r)=\left[4 \alpha^{2} r^{2}+\beta^{2}+4 \alpha \beta r+\frac{\lambda^{2}}{r^{2}}+\frac{4 \eta^{2}}{r^{2}+1}\right. \\
& -\frac{4 \eta^{2}}{\left(r^{2}+1\right)^{2}}+\frac{4 \lambda \eta}{r^{2}+1}+4 \alpha \lambda+8 \alpha \eta-\frac{8 \alpha \eta}{r^{2}+1}+\frac{2 \beta \lambda}{r} \\
& \left.+4 \beta \eta \frac{r}{r^{2}+1}+2 \alpha-\frac{\lambda}{r^{2}}-\frac{2 \eta}{r^{2}+1}+\frac{4 \eta}{\left(r^{2}+1\right)^{2}}\right] \\
& \cdot u_{\nu \gamma}(r)
\end{aligned}
$$

or

$$
\begin{aligned}
& u_{0, \gamma}^{\prime \prime}(r)=\left[4 \alpha^{2} r^{2}+\beta^{2}+4 \alpha \lambda+8 \alpha \eta+2 \alpha+4 \alpha \beta r\right. \\
& +\frac{\lambda^{2}}{r^{2}}-\frac{\lambda}{r^{2}}+\frac{2 \beta \lambda}{r}+\frac{4 \eta^{2}}{r^{2}+1}+\frac{4 \lambda \eta}{r^{2}+1}-\frac{2 \eta}{r^{2}+1} \\
& \left.-\frac{8 \alpha \eta}{r^{2}+1}-\frac{4 \eta^{2}}{\left(r^{2}+1\right)^{2}}+\frac{4 \eta}{\left(r^{2}+1\right)^{2}}+4 \beta \eta \frac{r}{r^{2}+1}\right] \\
& \cdot u_{0, \gamma}(r) .
\end{aligned}
$$

After some simplicity, by comparing (7) and (14), it can be found that

$$
\begin{aligned}
\lambda^{2}-\lambda-2 \mu d-\frac{(2 \gamma+5)(2 \gamma+3)}{4} & =0, \\
4 \alpha^{2} & =2 \mu a, \\
-4 \eta^{2}+4 \eta & =-2 \mu k, \\
4 \beta \eta & =2 \mu h, \\
4 \eta^{2}+4 \lambda \eta-8 \alpha \eta-2 \eta & =2 \mu k, \\
4 \alpha \beta & =2 \mu b, \\
2 \beta \lambda & =2 \mu c, \\
\beta^{2}+4 \alpha \lambda+8 \alpha \eta+2 \alpha & =-2 \mu E .
\end{aligned}
$$


Equation (15) immediately yields

$$
\begin{aligned}
& \lambda=\frac{1+\sqrt{1+8 \mu d+(2 \gamma+5)(2 \gamma+3)}}{2}, \\
& \alpha=-\sqrt{\frac{\mu a}{2}}, \\
& \eta=\frac{1+\sqrt{1+2 \mu k}}{2}, \\
& \beta=\frac{\mu h}{2 \eta}, \\
& a=\frac{(2 \lambda+1)^{2}}{8 \mu}, \\
& b=\frac{2 \alpha \beta}{\mu}, \\
& c=\frac{\beta \lambda}{\mu} .
\end{aligned}
$$

And the energy can be obtained by

$$
E_{\nu \gamma}=-\frac{1}{2 \mu}\left(\beta^{2}+4 \alpha \lambda+8 \alpha \eta+2 \alpha\right) .
$$

The spin and isospin dependent interactions are not the only source of $S U(6)$ violation. In order to study the baryon spectrum one has to consider $S U(3)$ violation produced by the differences in the quark masses. The Gell-Mann-Okubo (GMO) mass formula [54] made use of a $\lambda_{8}$ violation of $S U(3)$ in order to explain the mass splitting within the various $S U(3)$ multiplets. The hypercentral Constituent Quark Model is fairly good for description of the baryon spectrum [55], but in some cases the splitting within the various $S U(6)$ multiplets is too low. The preceding results $[35,54,55]$ show that both spin and isospin dependent terms in the quark Hamiltonian are important. Description of the splitting within $S U(6)$ baryon multiplets is presented by the Gürsey Radicati mass formula [36]:

$$
\begin{aligned}
M= & M_{0}+C C_{2}\left[S U_{S}(2)\right]+D C_{1}\left[U_{Y}(1)\right] \\
& +I\left[C_{2}\left[S U_{I}(2)\right]-\frac{1}{4}\left(C_{1}\left[U_{Y}(1)\right]\right)^{2}\right],
\end{aligned}
$$

where $M_{0}$ is the average energy value of $S U(6)$ multiplet, $C_{2}\left[S U_{S}(2)\right]$ and $C_{2}\left[S U_{I}(2)\right]$ are the $S U(2)$ Casimir operators for spin and isospin, respectively, and $C_{1}\left[U_{Y}(1)\right]$ is the Casimir operator for $U(1)$ subgroup generated by the hypercharge $Y$ [56-58]. This mass formula has been tested to be successful in the description of the ground state baryon masses; however, as stated by the authors themselves, it is not the most general mass formula that can be written on the basis of a broken $S U(6)$ symmetry. In order to generalize (18), Giannini et al. considered dynamical spin-flavor symmetry $S U_{\mathrm{SF}}(6)$ [43] and described $S U_{\mathrm{SF}}(6)$ symmetry breaking mechanism by generalizing (18) as

$$
\begin{aligned}
M= & M_{0}+A C_{2}\left[S U_{\mathrm{SF}}(6)\right]+B C_{2}\left[S U_{F}(3)\right] \\
& +C C_{2}\left[S U_{S}(2)\right]+D C_{1}\left[U_{Y}(1)\right] \\
& +I\left[C_{2}\left[S U_{I}(2)\right]-\frac{1}{4}\left(C_{1}\left[U_{Y}(1)\right]\right)^{2}\right] .
\end{aligned}
$$

TABLE 1: Eigenvalues of $C_{2}\left[S U_{S F}(6)\right]$ and $C_{2}\left[S U_{F}(3)\right]$ Casimir operators.

\begin{tabular}{lccc}
\hline Dimension $(S U(6))$ & $C_{2}\left[S U_{S F}(6)\right]$ & Dimension $(S U(3))$ & $C_{2}\left[S U_{F}(3)\right]$ \\
\hline 56 & $45 / 4$ & 8 & 3 \\
70 & $33 / 4$ & 10 & 6 \\
20 & $21 / 4$ & 1 & 0 \\
\hline
\end{tabular}

In (19) the spin term $\left(C C_{2}\left[S U_{S}(2)\right]\right)$ represents the spinspin interactions, the flavor term $\left(B C_{2}\left[S U_{F}(3)\right]\right)$ denotes the flavor dependence of the interactions, and the $S U_{\mathrm{SF}}(6)$ term $\left(A C_{2}\left[S U_{\mathrm{SF}}(6)\right]\right)$ depends on the permutation symmetry of the wave functions, representing "signature-dependent" interactions $[56,57]$. The last two terms $\left(I\left[C_{2}\left[S U_{I}(2)\right]-\right.\right.$ $\left.\left.(1 / 4)\left(C_{1}\left[U_{Y}(1)\right]\right)^{2}\right]\right)$ represent the isospin and hypercharge dependence of the masses. In Table 1, we give the expectation values of the Casimir operators $S U_{S F}(6)$ and $S U_{F}(3)$ for the allowed three-quark configurations.

The generalized Gürsey Radicati mass formula (19) can be used to describe the octet and decuplet baryons spectrum, provided that two conditions are fulfilled. The first condition is the feasibility of using the same splitting coefficients for different $S U(6)$ multiplets. This seems actually to be the case, as shown by the algebraic approach to the baryon spectrum [1]. The second condition is given by the feasibility of getting reliable values for the unperturbed mass values $M_{0}$ [32]. For this purpose we regarded $S U(6)$ invariant part of the hCQM which provides a good description of the baryon spectra and used the Gürsey Radicati inspired SU(6) breaking interaction to describe the splitting within each $S U$ (6) multiplet. Therefore, the baryons masses are obtained by three-quark masses and eigenenergies $\left(E_{\nu \gamma}\right)$ of the radial Schrödinger equation with the expectation values of $H_{\mathrm{GR}}$ as follows:

$$
M=3 m+E_{\nu \gamma}+\left\langle H_{\mathrm{GR}}\right\rangle .
$$

In the above equation $m$ is the reduced mass. $H_{\mathrm{GR}}$ is in the following form:

$$
\begin{aligned}
H_{\mathrm{GR}}= & A C_{2}\left[S U_{\mathrm{SF}}(6)\right]+B C_{2}\left[S U_{F}(3)\right] \\
& +C C_{2}\left[S U_{S}(2)\right]+D C_{1}\left[U_{Y}(1)\right] \\
& +I\left[C_{2}\left[S U_{I}(2)\right]-\frac{1}{4}\left(C_{1}\left[U_{Y}(1)\right]\right)^{2}\right] .
\end{aligned}
$$

The expectation values of $H_{\mathrm{GR}}\left(\left\langle H_{\mathrm{GR}}\right\rangle\right)$ are completely identified by the expectation values of the Casimir operators (Table 1).

Therefor we have

$$
\begin{aligned}
M= & 3 m+E_{\gamma \gamma}+A\left\langle C_{2}\left[S U_{\mathrm{SF}}(6)\right]\right\rangle \\
& +B\left\langle C_{2}\left[S U_{F}(3)\right]\right\rangle+C\left\langle C_{2}\left[S U_{S}(2)\right]\right\rangle \\
& +D\left\langle C_{1}\left[U_{Y}(1)\right]\right\rangle \\
& +I\left[\left\langle C_{2}\left[S U_{I}(2)\right]\right\rangle-\frac{1}{4}\left\langle\left(C_{1}\left[U_{Y}(1)\right]\right)\right\rangle^{2}\right] .
\end{aligned}
$$


TABLE 2: The fitted values of the parameters of (22) for $N, \Delta, \Lambda, \Sigma, \Xi$, and $\Omega$ baryons, obtained with resonances mass differences and global fit to the experimental resonance masses [42].

\begin{tabular}{lcccccccccc}
\hline Parameter & $A$ & $B$ & $C$ & $D$ & $I$ & $m$ & $\alpha$ & $\beta$ & $d$ & $\eta$ \\
\hline Value & $-19.616 \mathrm{MeV}$ & $18.575 \mathrm{MeV}$ & 38.3 & $-197.3 \mathrm{MeV}$ & $38.5 \mathrm{MeV}$ & $271 \mathrm{MeV}$ & $-0.381 \mathrm{MeV}^{2}$ & $0.489 \mathrm{MeV}$ & 0.388 & 0.446 \\
\hline
\end{tabular}

TABLE 3: Mass spectrum of baryons resonances (in MeV) calculated with the mass formula (22). The column $M_{\text {Our Calc }}$ contains our calculations with the parameters of Table 2 and column $M_{[43] \text { Calc }}$ shows calculations of Giannini et al. Column 7 indicates the percentage of relative error for our calculations.

\begin{tabular}{|c|c|c|c|c|c|c|}
\hline Baryon & Status & $M_{[42] \exp }$ & State & $M_{[43] \mathrm{Calc}}$ & $M_{\text {Our Calc }}$ & Percent of relative error for our calculation \\
\hline$N(938) P 11$ & $* * * *$ & 938 & ${ }^{2} 8_{1 / 2}\left[56,0^{+}\right]$ & 938 & 938 & $0 \%$ \\
\hline$N(1440) P 11$ & $* * * *$ & $1410-1450$ & ${ }^{2} 8_{1 / 2}\left[56,0^{+}\right]$ & 1448.7 & 1448.51 & $2.73 \%-0.1 \%$ \\
\hline$N(1520) D 13$ & $* * * *$ & $1510-1520$ & ${ }^{2} 8_{3 / 2}\left[70,1^{-}\right]$ & 1543.7 & 1528.79 & $1.24 \%-0.57 \%$ \\
\hline$N(1535) S 11$ & $* * * *$ & $1525-1545$ & ${ }^{2} 8_{1 / 2}\left[70,1^{-}\right]$ & 1543.7 & 1528.79 & $0.24 \%-1.04 \%$ \\
\hline$N(1650) S 11$ & $* * * *$ & $1645-1670$ & ${ }^{4} 8_{1 / 2}\left[70,1^{-}\right]$ & 1658.6 & 1643.69 & $0.07 \%-1.57 \%$ \\
\hline$N(1675) D 15$ & $* * * *$ & $1670-1680$ & ${ }^{4} 8_{5 / 2}\left[70,1^{-}\right]$ & 1658.6 & 1643.69 & $1.57 \%-2.16 \%$ \\
\hline$N(1680) F 15$ & $* * *$ & $1680-1690$ & ${ }^{2} 8_{5 / 2}\left[56,2^{+}\right]$ & 1651.4 & 1688.57 & $0.51 \%-0.08 \%$ \\
\hline$N(1700) D 13$ & $* * *$ & $1650-1750$ & ${ }^{4} 8_{3 / 2}\left[70,1^{-}\right]$ & 1658.6 & 1643.69 & $0.38 \%-6.07 \%$ \\
\hline$N(1710) P 11$ & $* * *$ & $1680-1740$ & ${ }^{2} 8_{1 / 2}\left[56,0^{+}\right]$ & 1795.4 & 1798.16 & $7.03 \%-3.34 \%$ \\
\hline$N(1720) P 13$ & $* * * *$ & $1700-1750$ & ${ }^{2} 8_{3 / 2}\left[56,2^{+}\right]$ & 1651.4 & 1688.57 & $0.67 \%-3.51 \%$ \\
\hline$N(1875) D 13$ & $* * *$ & $1820-1920$ & ${ }^{2} 8_{3 / 2}\left[70,1^{-}\right]$ & - & 1857.01 & $2.03 \%-3.28 \%$ \\
\hline$N(1900) P 13$ & $* * *$ & 1875-1935 & ${ }^{2} 8_{3 / 2}\left[70,2^{+}\right]$ & - & 1966.7 & $4.89 \%-1.63 \%$ \\
\hline$N(2190) G 17$ & $* * * *$ & $2100-2200$ & ${ }^{2} 8_{7 / 2}\left[70,3^{-}\right]$ & - & 2186.37 & $4.11 \%-0.61 \%$ \\
\hline$N(2220) H 19$ & $* * * *$ & $2200-2300$ & ${ }^{2} 8_{9 / 2}\left[56,4^{+}\right]$ & - & 2237.44 & $1.7 \%-2.72 \%$ \\
\hline$N(2250) G 19$ & $* * * *$ & $2200-2350$ & ${ }^{4} 8_{9 / 2}\left[70,3^{-}\right]$ & - & 2301.27 & $4.6 \%-2.07 \%$ \\
\hline$N(2600) I 1,11$ & $* * *$ & $2550-2750$ & ${ }^{2} 8_{11 / 2}\left[70,5^{-}\right]$ & - & 2626.25 & $2.99 \%-4.5 \%$ \\
\hline$\Delta(1232) P 33$ & $* * * *$ & $1230-1234$ & ${ }^{4} 10_{3 / 2}\left[56,0^{+}\right]$ & 1232 & 1232.37 & $0.19 \%-0.13 \%$ \\
\hline$\Delta(1600) P 33$ & $* * *$ & $1500-1700$ & ${ }^{4} 10_{3 / 2}\left[56,0^{+}\right]$ & 1683 & 1647.2 & $9.81 \%-3.1 \%$ \\
\hline$\Delta(1620) S 31$ & $* * * *$ & $1600-1660$ & ${ }^{2} 10_{1 / 2}\left[70,1^{-}\right]$ & 1722.8 & 1700.01 & $6.25 \%-2.41 \%$ \\
\hline$\Delta(1700) D 33$ & $* * * *$ & $1670-1750$ & ${ }^{2} 10_{3 / 2}\left[70,1^{-}\right]$ & 1722.8 & 1700.01 & $1.79 \%-2.85 \%$ \\
\hline$\Delta(1905) F 35$ & $* * * *$ & $1855-1910$ & ${ }^{4} 10_{5 / 2}\left[56,2^{+}\right]$ & 1945.4 & 1865.27 & $0.55 \%-2.34 \%$ \\
\hline$\Delta(1910) P 31$ & $* * * *$ & $1860-1910$ & ${ }^{4} 10_{1 / 2}\left[56,2^{+}\right]$ & 1945.4 & 1865.27 & $0.28 \%-2.34 \%$ \\
\hline$\Delta(1920) P 33$ & $* * *$ & $1900-1970$ & ${ }^{4} 10_{3 / 2}\left[56,0^{+}\right]$ & 2089.4 & 1974.7 & $3.93 \%-0.23 \%$ \\
\hline$\Delta(1930) D 35$ & $* * *$ & $1900-2000$ & ${ }^{2} 10_{5 / 2}\left[70,2^{-}\right]$ & - & 1918.6 & $0.97 \%-4.07 \%$ \\
\hline$\Delta(1950) D 35$ & $* * * *$ & 1915-1950 & ${ }^{4} 10_{7 / 2}\left[56,2^{+}\right]$ & 1945.4 & 1865.27 & $2.59 \%-4.34 \%$ \\
\hline$\Delta(2420) H 3,11$ & $* * * *$ & $2300-2500$ & ${ }^{4} 10_{11 / 2}\left[56,4^{+}\right]$ & - & 2303.79 & $0.16 \%-7.84 \%$ \\
\hline
\end{tabular}

${ }^{* * * *}$ Existence is certain, and properties are at least fairly well explored. ${ }^{* * *}$ Existence ranges from very likely to certain, but further confirmation is desirable and/or quantum numbers, branching fractions, and so forth are not well determined.

In order to simplify the solving procedure, the constituent quarks masses are assumed to be the same for Up, Down, and Strange quark flavors $\left(m=m_{u}=m_{d}=m_{s}\right)$; therefore, within this approximation, $S U(6)$ symmetry is only broken dynamically by the spin and flavor dependent terms in the Hamiltonian. We determined $E_{\gamma \gamma}$ by exact solution of the radial Schrödinger equation for the hypercentral potential equation (5). For calculating the baryons mass according to (22), we need to find the unknown parameters. For this purpose we choose a limited number of well-known resonances and express their mass differences using $H_{\mathrm{GR}}$ and the Casimir operator expectation values: $N(1650) S 11-N(1535) S 11=$ $3 C, 4 N(938) P 11-\Sigma(1193) P 11-3 \Lambda(1116) P 01=4 D$, and $\Sigma(1193) P 11-\Lambda(1116) P 01=2 I$, leading to the numerical values $C=38.3, D=-197.3 \mathrm{MeV}$, and $I=38.5 \mathrm{MeV}$. For determining $m, \alpha, \beta, d$, and $\eta$ (in (17)) and the two coefficients
$A$ and $B$ of (19) we have used the Newton-Raphson Method for solving the nonlinear equations. For our purpose we chose $N(938) P 11, \Delta(1232) P 33, \Lambda(1116) P 01, \Sigma(1193) P 11$, $\Lambda(1810) P 01, \Delta(1700) D 33$, and $\Sigma(1940) D 13$ which yielded the best reproduction (the maximum percentage of relative error is $0.33 \%$ ); then by solving seven nonlinear equations with seven unknown parameters we calculated the free parameters $(m, \alpha, \beta, d, \eta, A, B)$. The fitted parameters are reported in Table 2 . The corresponding numerical values for 3 and 4 star baryons resonances are given in Tables 3 and 4, column $M_{\text {our Calc }}$. In Tables 3 and 4, column $M_{[43] \text { Calc }}$, we have shown the numerical values of the calculated masses of baryon resonances by Giannini et al., where they regarded the confinement potential as the Cornell potential $(-(\tau / x)+$ $\alpha x)$. The solution of the hypercentral Schrödinger equation with this potential cannot be obtained analytically [43]; 
TABLE 4: Like Table 3, but for $\Lambda, \Sigma$, $\Xi$, and $\Omega$ resonances.

\begin{tabular}{|c|c|c|c|c|c|c|}
\hline Baryon & Status & $M_{[42] \exp }$ & State & $M_{[43] \text { Calc }}$ & $M_{\text {Our Calc }}$ & Percent of relative error for our calculation \\
\hline$\Lambda(1116) P 01$ & $* * * *$ & 1116 & ${ }^{2} 8_{1 / 2}\left[56,0^{+}\right]$ & 1116 & 1116.05 & $0.004 \%$ \\
\hline$\Lambda(1600) P 01$ & $* * *$ & $1560-1700$ & ${ }^{2} 8_{1 / 2}\left[56,0^{+}\right]$ & 1626.7 & 1647.99 & $5.64 \%-3.05 \%$ \\
\hline$\Lambda(1670) S 01$ & $* * * *$ & $1660-1680$ & ${ }^{2} 8_{1 / 2}\left[70,1^{-}\right]$ & 1721.7 & 1706.84 & $2.82 \%-1.59 \%$ \\
\hline$\Lambda(1690) D 03$ & $* * * *$ & $1685-1695$ & ${ }^{2} 8_{3 / 2}\left[70,1^{-}\right]$ & 1721.7 & 1706.84 & $1.29 \%-0.69 \%$ \\
\hline$\Lambda(1800) S 01$ & $* * *$ & $1720-1850$ & ${ }^{4} 8_{1 / 2}\left[70,1^{-}\right]$ & 1836.6 & 1821.74 & $5.91 \%-1.52 \%$ \\
\hline$\Lambda(1810) P 01$ & $* * *$ & $1750-1850$ & ${ }^{2} 8_{1 / 2}\left[70,0^{+}\right]$ & 1973.4 & 1816.04 & $3.77 \%-1.83 \%$ \\
\hline$\Lambda(1820) F 05$ & $* * * *$ & $1815-1825$ & ${ }^{2} 8_{5 / 2}\left[56,2^{+}\right]$ & 1829.4 & 1866.62 & $2.84 \%-2.28 \%$ \\
\hline$\Lambda(1830) D 05$ & $* * * *$ & $1810-1830$ & ${ }^{4} 8_{5 / 2}\left[70,1^{-}\right]$ & 1836.6 & 1821.74 & $0.64 \%-0.45 \%$ \\
\hline$\Lambda(1890) P 03$ & $* * * *$ & $1850-1910$ & ${ }^{2} 8_{3 / 2}\left[56,2^{+}\right]$ & 1829.4 & 1866.62 & $0.89 \%-2.27 \%$ \\
\hline$\Lambda(2100) G 07$ & $* * * *$ & $2090-2110$ & ${ }^{2} 1_{7 / 2}\left[70,3^{-}\right]$ & - & 2089.04 & $0.04 \%-0.99 \%$ \\
\hline$\Lambda(2110) F 05$ & $* * * *$ & $2090-2140$ & ${ }^{4} 8_{5 / 2}\left[70,2^{+}\right]$ & 1995 & 2149.96 & $2.86 \%-0.46 \%$ \\
\hline$\Lambda(2350) H 09$ & $* * *$ & $2340-2370$ & ${ }^{2} 8_{9 / 2}\left[56,4^{+}\right]$ & - & 2360.52 & $0.87 \%-0.4 \%$ \\
\hline$\Lambda^{*}(1405) S 01$ & $* * * *$ & $1402-1410$ & ${ }^{2} 1_{1 / 2}\left[70,1^{-}\right]$ & 1657.9 & 1433.91 & $2.27 \%-1.69 \%$ \\
\hline$\Lambda^{*}(1520) D 01$ & $* * * *$ & $1518-1520$ & ${ }^{2} 1_{3 / 2}\left[70,1^{-}\right]$ & 1657.9 & 1433.91 & $5.53 \%-5.66 \%$ \\
\hline$\Sigma(1193) P 11$ & $* * * *$ & 1193 & ${ }^{2} 8_{1 / 2}\left[56,0^{+}\right]$ & 1193 & 1193.05 & $0.004 \%$ \\
\hline$\Sigma(1660) P 11$ & $* * *$ & $1630-1690$ & ${ }^{2} 8_{1 / 2}\left[56,0^{+}\right]$ & 1703.7 & 1616.12 & $0.05 \%-4.37 \%$ \\
\hline$\Sigma(1670) D 13$ & $* * * *$ & $1665-1685$ & ${ }^{2} 8_{3 / 2}\left[70,1^{-}\right]$ & 1798.7 & 1783.74 & $7.13 \%-5.85 \%$ \\
\hline$\Sigma(1750) S 11$ & $* * *$ & $1730-1800$ & ${ }^{2} 8_{1 / 2}\left[70,1^{-}\right]$ & 1798.7 & 1783.74 & $3.1 \%-0.9 \%$ \\
\hline$\Sigma(1775) D 15$ & $* * * *$ & $1770-1780$ & ${ }^{4} 8_{5 / 2}\left[70,1^{-}\right]$ & 1913.6 & 1789.87 & $1.12 \%-0.55 \%$ \\
\hline$\Sigma(1915) F 15$ & $* * * *$ & 1900-1935 & ${ }^{2} 8_{5 / 2}\left[56,2^{+}\right]$ & 1906.4 & 1910.7 & $0.56 \%-1.25 \%$ \\
\hline$\Sigma(1940) D 13$ & $* * *$ & 1900-1950 & ${ }^{2} 8_{3 / 2}\left[56,1^{-}\right]$ & 1913.6 & 1943.62 & $2.29 \%-0.32 \%$ \\
\hline$\Sigma^{*}(1385) P 13$ & $* * * *$ & 1383-1385 & ${ }^{4} 10_{3 / 2}\left[56,0^{+}\right]$ & - & 1363.67 & $1.39 \%-1.54 \%$ \\
\hline$\Sigma^{*}(2030) F 17$ & $* * * *$ & $2025-2040$ & ${ }^{4} 10_{7 / 2}\left[56,2^{+}\right]$ & 2085.0 & 2004.82 & $0.99 \%-1.72 \%$ \\
\hline$\Xi(1318) P 11$ & $* * * *$ & $1314-1316$ & ${ }^{2} 8_{1 / 2}\left[56,0^{+}\right]$ & 1332.6 & 1332.6 & $1.41 \%-1.26 \%$ \\
\hline$\Xi(1690) S 11$ & $* * *$ & $1680-1700$ & ${ }^{2} 8_{1 / 2}\left[70,1^{-}\right]$ & - & 1706.1 & $1.55 \%-0.35 \%$ \\
\hline$\Xi(1820) D 13$ & $* * *$ & $1818-1828$ & ${ }^{2} 8_{3 / 2}\left[70,1^{-}\right]$ & 1938.3 & 1923.39 & $5.79 \%-5.21 \%$ \\
\hline$\Xi^{*}(1530) P 13$ & $* * * *$ & $1531-1532$ & ${ }^{4} 10_{3 / 2}\left[56,0^{+}\right]$ & 1511.1 & 1503.2 & $1.81 \%-1.87 \%$ \\
\hline$\Omega(1672) P 03$ & $* * * *$ & $1672-1673$ & ${ }^{4} 10_{3 / 2}\left[56,0^{+}\right]$ & 1650.7 & 1643 & $1.73 \%-1.79 \%$ \\
\hline$\Omega(2250) D 03$ & $* * *$ & $2243-2261$ & ${ }^{2} 10_{3 / 2}\left[70,1^{-}\right]$ & - & 2227.87 & $0.67 \%-1.46 \%$ \\
\hline
\end{tabular}

${ }^{* * * *}$ Existence is certain, and properties are at least fairly well explored. ${ }^{* * *}$ Existence ranges from very likely to certain, but further confirmation is desirable and/or quantum numbers, branching fractions, and so forth are not well determined.

therefore Giannini et al. used the dynamic symmetry $O(7)$ of the hyperCoulomb problem to obtain the hyperCoulomb Hamiltonian and eigenfunctions analytically and also they regarded the linear term as a perturbation. Comparison between our results and the experimental masses [42] shows that our model has improved the results of model in [43], particularly in $\Lambda(1810), \Lambda(2110) F 05, \Lambda^{*}(1405) S 01$, $\Lambda^{*}(1520) D 01, \Delta(1905) F 35, \Delta(1910) P 31, \Delta(1920) P 33$, and $\Sigma(1775) D 15$ (refer to Tables 3 and 4). These improvements in reproduction of baryons resonance masses are obtained by using a suitable form for confinement potential and exact analytical solution of the radial Schrödinger equation for our proposed potential. The percentage of relative error for our calculations is between 0 and $10 \%$ (column 7, in Tables 3 and 4). The corresponding numerical values for some of 1 and 2 star baryons resonances mass up to $2.1 \mathrm{GeV}$ are given in Table 5, column $M_{\text {our Calc }}$. The percentage of relative error for our calculations is between 0.07 and $9 \%$ (column 6 , in Table 5). Comparison between our results and the experimental masses [42] shows that the baryon spectra are, in general, fairly well reproduced.

\section{Conclusion}

In this paper we have computed the baryon resonances spectrum up to $3 \mathrm{GeV}$ within a nonrelativistic quark model based on the three identical quarks Schrödinger equation and the algebraic approach. We have solved the Schrodinger equation numerically to obtain the energy eigenvalues under the Killingbeck plus isotonic oscillator interaction potentials. Then, we fitted the generalized GR mass formula parameters to the baryons energies and calculated the baryon masses. The overall good description of the spectrum which we obtain by our proposed model shows that our theoretical model can also be used to give a fair description of the energies of the excited multiplets up to $3 \mathrm{GeV}$ and not only for the ground state octets but also decuplets. Moreover, our model reproduces the position of the Roper resonances of the nucleon and negative-parity resonance. There are problems in the reproduction of the experimental masses in $\Delta(1620) S 31$ and $\Sigma(1670) D 13$ that turn out to have predicted mass about $100 \mathrm{MeV}$ above the experimental value. A better agreement may be obtained either using the square of the mass [1] or 
TABLE 5: Mass spectrum of some of 1 and 2 star baryons resonances (in $\mathrm{MeV}$ ) up to $2.1 \mathrm{GeV}$ calculated with the mass formula (22). column $M_{\text {Our Calc }}$ contains our calculations with the parameters of Table 2 and column 6 indicates the percentage of relative error for our calculations.

\begin{tabular}{|c|c|c|c|c|c|}
\hline Baryon & Status & $M_{[42] \exp }$ & State & $M_{\text {Our Calc }}$ & Percent of relative error for our calculation \\
\hline$N(1860) F 15$ & $* *$ & $1820-1960$ & ${ }^{2} 8_{5 / 2}\left[70,2^{+}\right]$ & 1966.7 & $8.06 \%-0.34 \%$ \\
\hline$N(1880) P 11$ & $* *$ & $1835-1905$ & ${ }^{4} 8_{1 / 2}\left[70,2^{+}\right]$ & 1971.9 & $7.46 \%-3.51 \%$ \\
\hline$N(1895) S 11$ & $* *$ & 1880-1910 & ${ }^{2} 8_{1 / 2}\left[70,1^{-}\right]$ & 1857.01 & $1.22 \%-2.77 \%$ \\
\hline$N(1990) F 17$ & $* *$ & $1995-2125$ & ${ }^{4} 8_{7 / 2}\left[70,2^{+}\right]$ & 1971.9 & $1.15 \%-7.2 \%$ \\
\hline$N(2000) F 15$ & $* *$ & $1950-2150$ & ${ }^{4} 8_{5 / 2}\left[70,2^{+}\right]$ & 1971.9 & $1.12 \%-8.28 \%$ \\
\hline$N(2040) P 13$ & * & 2031-2065 & ${ }^{4} 8_{3 / 2}\left[70,2^{+}\right]$ & 1971.9 & $2.9 \%-4.5 \%$ \\
\hline$N(2060) D 15$ & $* *$ & $2045-2075$ & ${ }^{4} 8_{5 / 2}\left[70,2^{-}\right]$ & 1971.9 & $3.57 \%-4.96 \%$ \\
\hline$N(2120) D 13$ & $* *$ & $2090-2210$ & ${ }^{2} 8_{3 / 2}\left[56,1^{-}\right]$ & 2127.52 & $1.79 \%-3.73 \%$ \\
\hline$\Delta(1750) P 31$ & * & $1708-1780$ & ${ }^{2} 10_{1 / 2}\left[70,0^{+}\right]$ & 1754.5 & $2.72 \%-1.43 \%$ \\
\hline$\Delta(1900) S 31$ & $* *$ & $1840-1920$ & ${ }^{2} 10_{1 / 2}\left[70,1^{-}\right]$ & 1918.6 & $4.27 \%-0.07 \%$ \\
\hline$\Delta(1940) D 33$ & $* *$ & 1940-2060 & ${ }^{2} 10_{3 / 2}\left[70,1^{-}\right]$ & 1918.6 & $1.1 \%-6.86 \%$ \\
\hline$\Delta(2000) F 35$ & $* *$ & $\approx 2000$ & ${ }^{2} 10_{5 / 2}\left[70,2^{+}\right]$ & 2028.2 & $1.41 \%$ \\
\hline$\Sigma(1580) D 13$ & * & $\approx 1580$ & ${ }^{4} 8_{3 / 2}\left[70,1^{-}\right]$ & 1574.12 & $0.37 \%$ \\
\hline$\Sigma(1620) S 11$ & $* *$ & $\approx 1620$ & ${ }^{2} 8_{1 / 2}\left[70,0^{-}\right]$ & 1674.97 & $3.39 \%$ \\
\hline$\Sigma(1770) P 11$ & * & $\approx 1770$ & ${ }^{2} 8_{1 / 2}\left[70,0^{+}\right]$ & 1783.84 & $0.78 \%$ \\
\hline$\Sigma(1880) P 11$ & $* *$ & $\approx 1880$ & ${ }^{2} 8_{1 / 2}\left[20,1^{+}\right]$ & 1842.68 & $1.98 \%$ \\
\hline$\Sigma(2000) S 11$ & * & $\approx 2000$ & ${ }^{2} 8_{1 / 2}\left[70,1^{-}\right]$ & 2002.47 & $0.12 \%$ \\
\hline$\Sigma(2070) F 15$ & $*$ & $\approx 2070$ & ${ }^{4} 8_{5 / 2}\left[70,2^{+}\right]$ & 2117.37 & $2.28 \%$ \\
\hline$\Sigma^{*}(1840) P 13$ & * & $\approx 1840$ & ${ }^{4} 10_{3 / 2}\left[56,0^{+}\right]$ & 1895.6 & $3.02 \%$ \\
\hline$\Sigma^{*}(2080) P 13$ & $* *$ & $\approx 2080$ & ${ }^{2} 10_{3 / 2}\left[70,2^{+}\right]$ & 2058.2 & $1.04 \%$ \\
\hline
\end{tabular}

** Evidence of existence is only fair. ${ }^{*}$ Evidence of existence is poor.

trying to include a spatial dependence in the $S U(6)$ breaking part.

\section{Competing Interests}

The author declares that they have no competing interests.

\section{References}

[1] R. Bijker, F. Iachello, and A. Leviatan, "Algebraic models of hadron structure. I. Nonstrange baryons," Annals of Physics, vol. 236, no. 1, pp. 69-116, 1994.

[2] E. Santopinto and M. M. Giannini, "Systematic study of longitudinal and transverse helicity amplitudes in the hypercentral constituent quark model," Physical Review C, vol. 86, Article ID 065202, 2012.

[3] B. Chakrabartiy, A. Bhattacharyaz, S. Mani, and A. Sagari, "Baryons in diquark-quark model," Acta Physica Polonica B, vol. 41, no. 1, pp. 95-101, 2010.

[4] S. Aoki, G. Boyd, R. Burkhalter et al., "Quenched light Hadron spectrum," Physical Review Letters, vol. 84, no. 2, pp. 238-241, 2000.

[5] L. I. Abou-Salem, "Study of baryon spectroscopy using a new potential form," Advances in High Energy Physics, vol. 2014, Article ID 196484, 5 pages, 2014.

[6] N. Salehi, H. Hassanabadi, and A. A. Rajabi, "The light and strange baryon spectrum in a non-relativistic hypercentral quark potential model and algebraic framework," European Physical Journal Plus, vol. 128, article 27, 2013.
[7] N. Salehi, H. Hassanabadi, and A. A. Rajabi, "Ground states and excitation spectra of baryons in a non-relativistic model with the anharmonic potential," Chinese Physics $C$, vol. 37, no. 11, Article ID 113101, 2013.

[8] M. M. Giannini and E. Santopinto, "The hypercentral constituent quark model and its application to baryon properties," Chinese Journal of Physics, vol. 53, Article ID 020301, 81 pages, 2015.

[9] M. Gell-Mann and Y. Ne'eman, The Eightfold Way, Frontiers in Physics, Benjamin, New York, NY, USA, 1964.

[10] J. J. J. Kokkedee, The Quark Model, Frontiers in Physics, Benjamin, New York, NY, USA, 1969, based on a series oflectures given at CERN in Autumn 1967.

[11] S. Narison, QCD as a Theory of Hadrons: From Partons to Confinement, Cambridge University Press, Cambridge, UK, 2004.

[12] F. J. Ynduráin, The Theory of Quark and Gluon Interactions, Texts and monographs in physics, Springer, Berlin, Germany, 3rd edition, 1999.

[13] A. M. Abazov, B. Abbott, M. Abolins et al., "Direct observation of the strange $b$ baryon $\Xi_{\bar{b}}$," Physical Review Letters, vol. 99, Article ID 052001, 2007.

[14] T. Aaltonen, A. Abulencia, J. Adelman et al., "Observation and mass measurement of the baryon $\Xi_{b}^{-}$," Physical Review Letters, vol. 99, no. 5, Article ID 052002, 7 pages, 2007.

[15] K. C. Bowler, R. D. Kenway, O. Oliveira et al., "Heavy baryon spectroscopy from the lattice," Physical Review D, vol. 54, no. 4, pp. 3619-3633, 1996. 
[16] R. Lewis, N. Mathur, and R. M. Woloshyn, "Charmed baryons in lattice QCD," Physical Review D, vol. 64, no. 9, Article ID 094509, 9 pages, 2001.

[17] N. Mathur, R. Lewis, and R. M. Woloshyn, "Charmed and bottom baryons from lattice nonrelativistic QCD," Physical Review D, vol. 66, Article ID 014502, 2002.

[18] S. Gottlieb and S. Tamhankar, "A lattice study of $\Lambda_{b}$ semileptonic decay," Nuclear Physics B-Proceedings Supplements, vol. 119, pp. 644-646, 2003.

[19] A. Ali Khan, T. Bhattacharya, S. Collins et al., "Heavy-light mesons and baryons with $b$ quarks," Physical Review D, vol. 62, no. 5, Article ID 054505, 17 pages, 2000.

[20] J. M. Flynn, F. Mescia, and A. S. Bin Tariq, "Spectroscopy of doubly-charmed baryons in lattice QCD," Journal of High Energy Physics, vol. 2003, no. 7, article 66, 2003.

[21] H. Na and S. Gottlieb, "Heavy baryon mass spectrum from lattice QCD with 2+1 flavors," http://arxiv.org/abs/hep-lat/ 0610009.

[22] H. Na and S. Gottlieb, "Charm and bottom heavy baryon mass spectrum from lattice QCD with 2+1," PoSLAT2007:124, https://arxiv.org/abs/0710.1422.

[23] R. Lewis and R. M. Woloshyn, "Bottom baryons from a dynamical lattice QCD simulation," Physical Review D, vol. 79, no. 1, Article ID 014502, 9 pages, 2009.

[24] D. M. Asner, I. I. Bigi, J. Charles et al., "Charm physics," International Journal of Modern Physics A, vol. 24, pp. 499-502, 2009.

[25] K. G. Wilson, "Confinement of quarks," Physical Review D, vol. 10, no. 8, pp. 2445-2459, 1974.

[26] S. Weinberg, "The problem of mass," Transactions of the New York Academy of Sciences, vol. 38, no. 1, pp. 185-201, 1977.

[27] S. Aoki, "Unquenched QCD simulation results," Nuclear Physics B-Proceedings Supplements, vol. 94, no. 1-3, pp. 3-18, 2001.

[28] D. Toussaint, "Spectrum results with Kogut-Susskind quarks," Nuclear Physics B (Proceedings Supplements), vol. 106-107, pp. 111-116, 2002.

[29] T. Kaneko, "Light hadron spectrum and quark masses," Nuclear Physics B-Proceedings Supplements, vol. 106, pp. 133-141, 2002.

[30] S. Aoki, G. Boyd, R. Burkhalter et al., "Quenched light hadron spectrum," Physical Review Letters, vol. 84, no. 2, pp. 238-241, 2000.

[31] S. Aoki, G. Boyd, R. Burkhalter et al., "Light hadron spectrum and quark masses from quenched lattice QCD," Physical Review D, vol. 67, no. 3, Article ID 034503, 46 pages, 2003.

[32] S. S. Bali, B. Bolder, N. Eicker et al., "Static potentials and glueball masses from QCD simulations with Wilson sea quarks," Physical Review D, vol. 62, Article ID 054503, 2000.

[33] C. Alexandrou, P. de Forcrand, and O. Jahn, “The ground state of three quarks," Nuclear Physics B-Proceedings Supplements, vol. 119, pp. 667-669, 2003.

[34] E. Santopinto, F. Iachello, and M. M. Giannini, "Nucleon form factors in a simple three-body quark model," The European Physical Journal A, vol. 1, no. 3, pp. 307-315, 1998.

[35] M. M. Giannini, E. Santopinto, and A. Vassallo, "Hypercentral constituent quark model and isospin dependence," The European Physical Journal A, vol. 12, no. 4, pp. 447-452, 2001.

[36] F. Gürsey and L. A. Radicati, "Spin and unitary spin independence of strong interactions," Physical Review Letters, vol. 13, pp. 173-175, 1964.
[37] N. Salehi and A. A. Rajabi, "Proton static properties by using hypercentral constituent quark model and isospin," Modern Physics Letters A, vol. 24, no. 32, pp. 2631-2637, 2009.

[38] N. Salehi and A. A. Rajabi, "A new method for obtaining the spectrum of baryon resonances in the Killingbeck potential," Physica Scripta, vol. 85, no. 5, Article ID 055101, 2012.

[39] N. Saad, R. L. Hall, H. Çiftçi, and Ö. Yesiltas, "Study of the generalized quantum isotonic nonlinear oscillator potential," Advances in Mathematical Physics, vol. 2011, Article ID 750168, 20 pages, 2011.

[40] R. L. Hall, N. Saad, and O. Yeşiltaş, "Generalized quantum isotonic nonlinear oscillator in $d$ dimensions," Journal of Physics A: Mathematical and Theoretical, vol. 43, no. 46, Article ID 465304, 2010.

[41] D. Agboola, J. Links, I. Marquette, and Y.-Z. Zhang, "New quasi-exactly solvable class of generalized isotonic oscillators," Journal of Physics A: Mathematical and Theoretical, vol. 47, no. 39, Article ID 395305, 17 pages, 2014.

[42] K. A. Olive, K. Agashe, C. Amsler et al., "Review of particle physics," Chinese Physics C, vol. 38, no. 9, Article ID 090001, 2014.

[43] M. M. Giannini, E. Santopinto, and A. Vassallo, "A new application of the Gürsey and Radicati mass formula," The European Physical Journal A, vol. 25, no. 2, pp. 241-247, 2005.

[44] N. Salehi, H. Hassanabadi, and A. A. Rajabi, "The light and strange baryon spectrum in a non-relativistic hypercentral quark potential model and algebraic framework," The European Physical Journal Plus, vol. 128, no. 3, article 27, pp. 1-9, 2013.

[45] K. Zalewski, "Nonrelativistic description of heavy quarkonia," Acta Physica Polonica B, vol. 29, no. 9, pp. 2535-2538, 1998.

[46] Y.-B. Ding, X.-Q. Li, and P.-N. Shen, "Variational estimation of the wave function at the origin for heavy quarkonium," Physical Review D, vol. 60, Article ID 074010, 1999.

[47] M. Znojil, "The generalized continued fractions and potentials of the Lennard-Jones type," Journal of Mathematical Physics, vol. 31, pp. 1955-1961, 1990.

[48] S. Zaim, H. Guelmamene, and A. Bahache, "2D Schrödinger equation with mixed potential in noncommutaive complex space," http://arxiv.org/abs/1410.0399v1.

[49] M. Znojil, "The generalized continued fractions and potentials of the Lennard-Jones type," Journal of Mathematical Physics, vol. 31, no. 8, pp. 1955-1961, 1990.

[50] A. A. Rajabi, "Static properties of hadrons from MIT bag model with interacting quarks," Indian Journal of Pure and Applied Physics, vol. 41, no. 2, pp. 89-94, 2003.

[51] A. A. Rajabi and N. Salehi, "Mesons states and their dependence on spin and isospin," Iranian Journal of Physics Research, vol. 8, no. 3, pp. 169-175, 2008.

[52] N. Salehi and H. Hassanabadi, "A new model for calculating the masses of light baryons resonances under the decatic potential," Romanian Reports in Physics, vol. 67, no. 2, pp. 403-411, 2015.

[53] N. Salehi and H. Hassanabadi, "Spectrum of light and strange baryon resonances under the decatic potential," International Journal of Modern Physics E, vol. 24, no. 1, Article ID 1550002, 10 pages, 2015.

[54] M. Gell-Mann and Y. Ne'eman, The Eightfold Way, W.A. Benjamin, New York, NY, USA, 1964.

[55] M. Ferraris, M. M. Giannini, M. Pizzo, E. Santopinto, and L. Tiator, "A three-body force model for the baryon spectrum," Physics Letters B, vol. 364, no. 4, pp. 231-238, 1995. 
[56] F. J. Squires, "On the continuation of partial-wave amplitudes to complex l," Il Nuovo Cimento, vol. 25, no. 2, pp. 242-253, 1962.

[57] A. Martin, "Remarks on the prolongation of a scattering amplitude in the complex angular momentum plane," Physics Letters. B, vol. 1, pp. 72-75, 1962.

[58] R. Bijker, M. M. Giannini, and E. Santopinto, "Spectroscopy of pentaquark states," The European Physical Journal A, vol. 22, no. 2, pp. 319-329, 2004. 

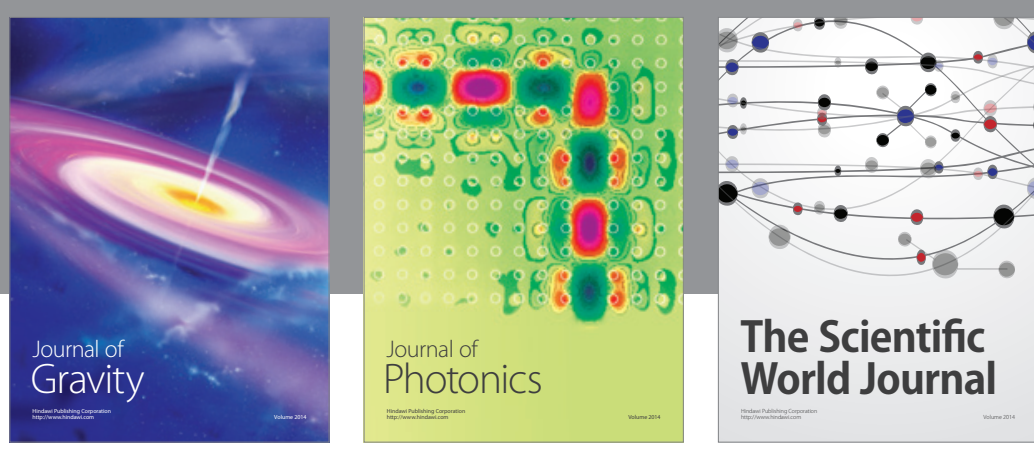

The Scientific World Journal
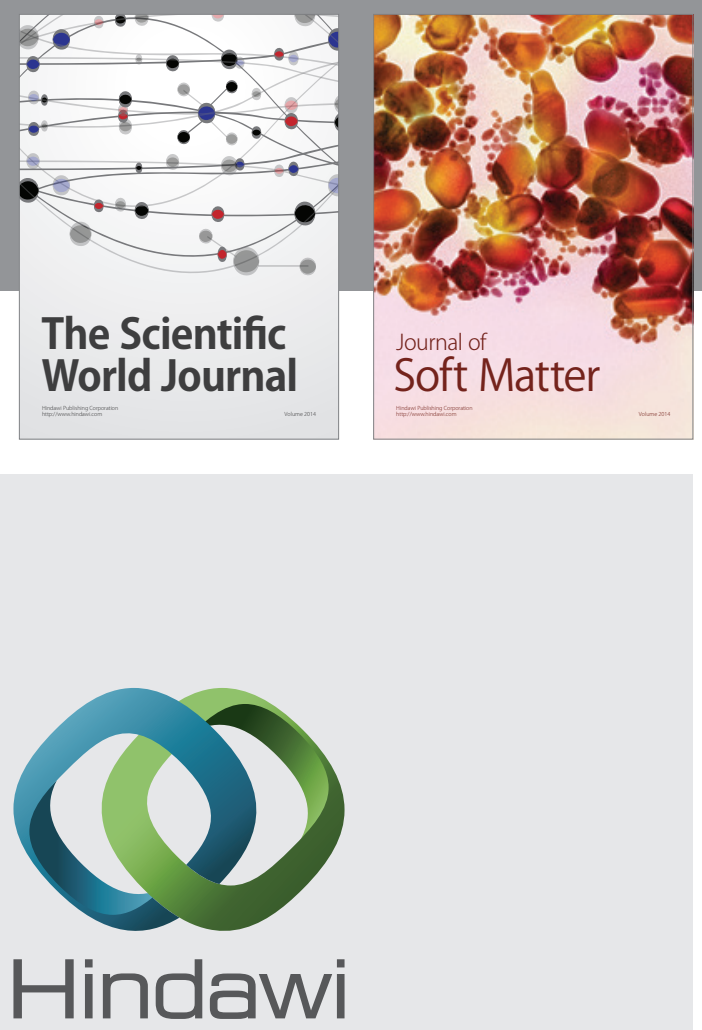

Submit your manuscripts at

http://www.hindawi.com

nternational Journal of

Statistical Mechanics
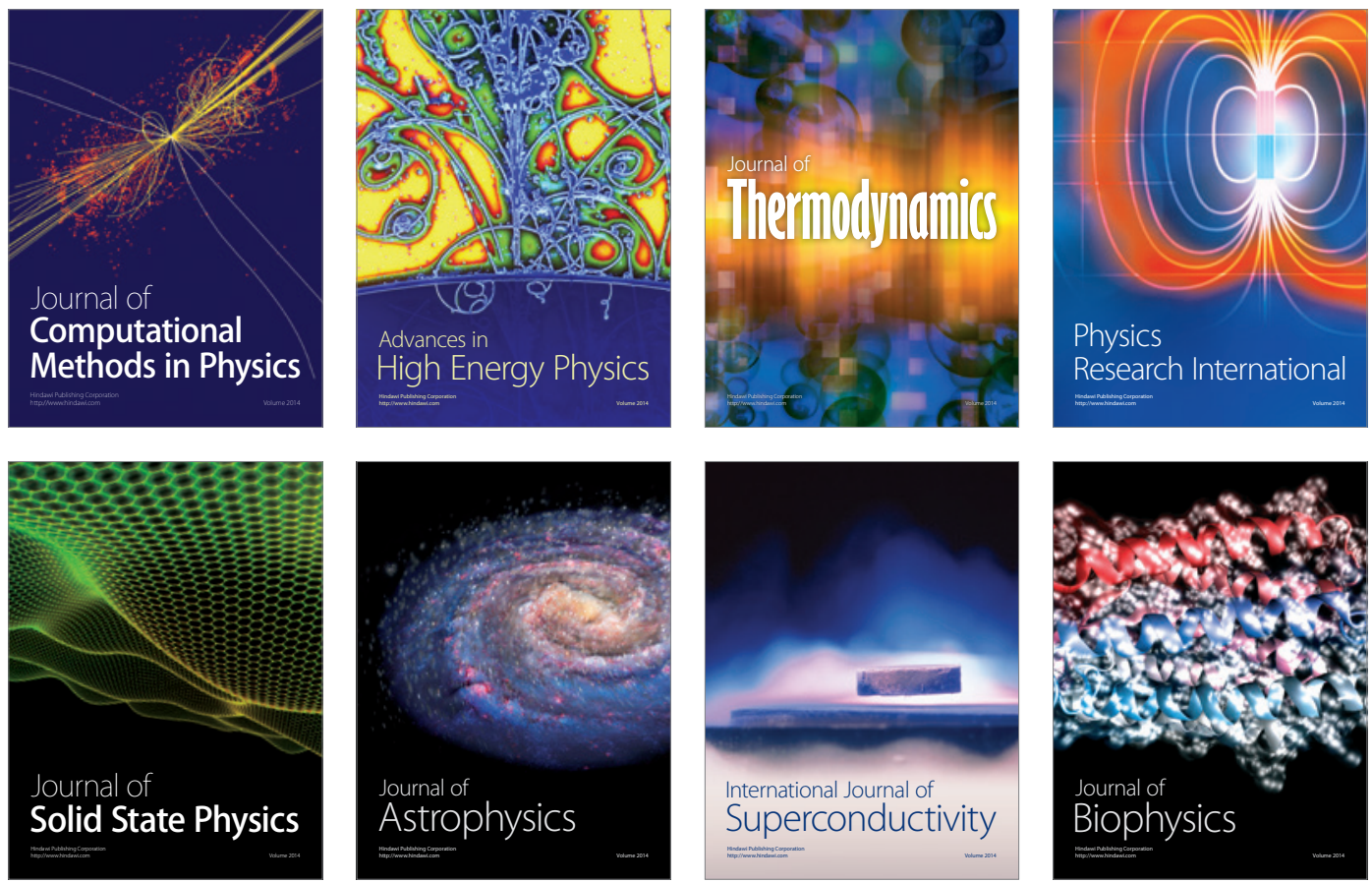
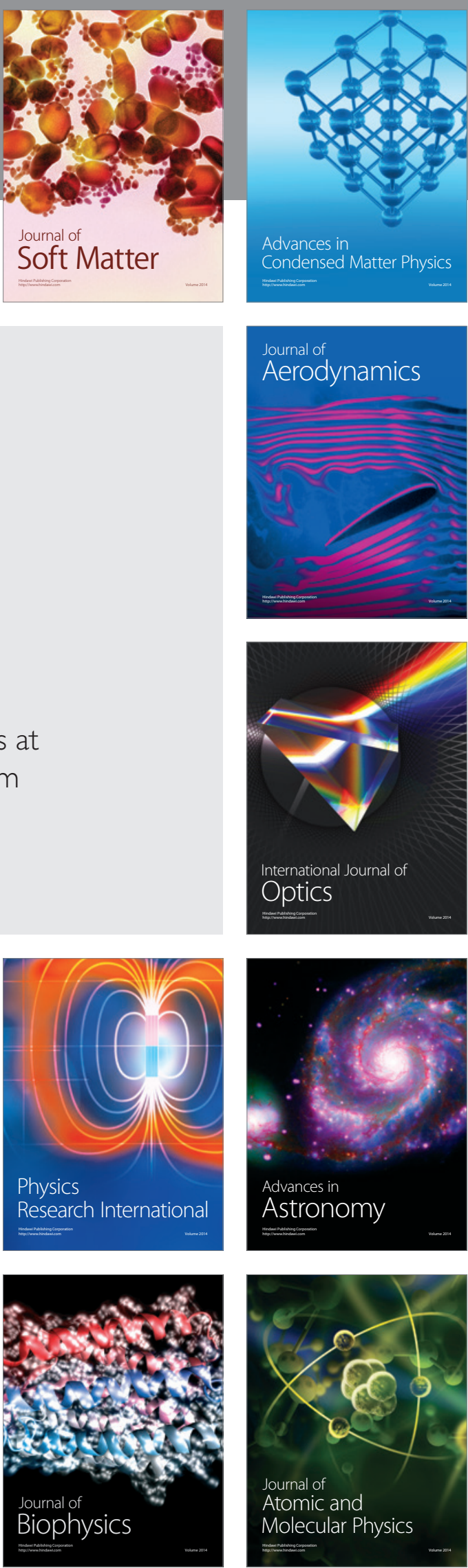